\title{
Discovery of Semantic Relationships in PolSAR Images Using Latent Dirichlet Allocation
}

\author{
Radu Tănase, Reza Bahmanyar, Gottfried Schwarz, and Mihai Datcu, Fellow, IEEE
}

\begin{abstract}
We propose a multi-level semantics discovery approach for bridging the semantic gap when mining highresolution Polarimetric Synthetic Aperture Radar (PolSAR) remote sensing images. First, an Entropy/Anisotropy/Alpha-Wishart classifier is employed to discover low-level semantics as classes representing the physical scattering properties of targets (e.g., low-entropy/surface-scattering/high-anisotropy). Then, the images are tiled into patches and each patch is modeled as a Bag-of-Words (BoW), a histogram of the class labels. Next, Latent Dirichlet Allocation is applied to discover their higher-level semantics as a set of topics. Our results demonstrate that topic semantics are close to human semantics used for basic land-cover types (e.g., grassland). Therefore, using the topic description (Bag-of-Topics) of PolSAR images leads to a narrower semantic gap in image mining. Additionally, a visual exploration of the topic descriptions helps to find semantic relationships which can be used for defining new semantic categories (e.g., mixed landcover types) and designing rule-based categorization schemes.
\end{abstract}

Index Terms-PolSAR, Entropy/Anisotropy/Alpha-Wishart classification, Bag-of-Words, Latent Dirichlet Allocation, Bag-ofTopics, Semantic relationships.

\section{INTRODUCTION}

$\mathbf{T}$ HE multitude of modern remote sensing sensors allows us to analyze tremendous amounts of high-resolution Earth Observation (EO) images. Therefore, developing new Content-Based Image Retrieval (CBIR) systems being able to extract user-desired information from existing image databases is highly demanded. In the state-of-the-art literature, various CBIR systems have been proposed for EO image mining such as $\mathrm{I}^{3} \mathrm{KR}$ [1], KIM [2], and its accelerated variant [3]. For the existing systems, semantic image interpretations are usually provided through either manual image annotation or user acceptance (in active learning scenarios), which require much human effort and time, and bias the systems towards user perspectives [4]. Moreover, due to the differences between human image understanding and how computers interpret and process them (the so-called semantic gap [4], [5]), many image mining results provided by computers are still unsatisfactory.

In this letter, we propose a multi-level semantics discovery approach for bringing computer interpretation of a particular

We acknowledge that all authors contributed equally to this paper.

R. Tănase is with the Research Centre for Spatial Information, University Politehnica of Bucharest - CEOSpaceTech, Bucharest, Romania, and also with the Military Technical Academy, Bucharest, Romania (e-mail: radu_tns@yahoo.com).

R. Bahmanyar, G. Schwarz, and M. Datcu are with the Remote Sensing Technology Institute (IMF), German Aerospace Center (DLR), Wessling, Germany (e-mail: reza.bahmanyar@dlr.de; gottfried.schwarz@dlr.de; mihai.datcu@dlr.de).

M. Datcu is also with the Research Centre for Spatial Information of the University Politehnica of Bucharest - CEOSpaceTech, Bucharest, Romania.

Manuscript received July 2016; revised October 2016.

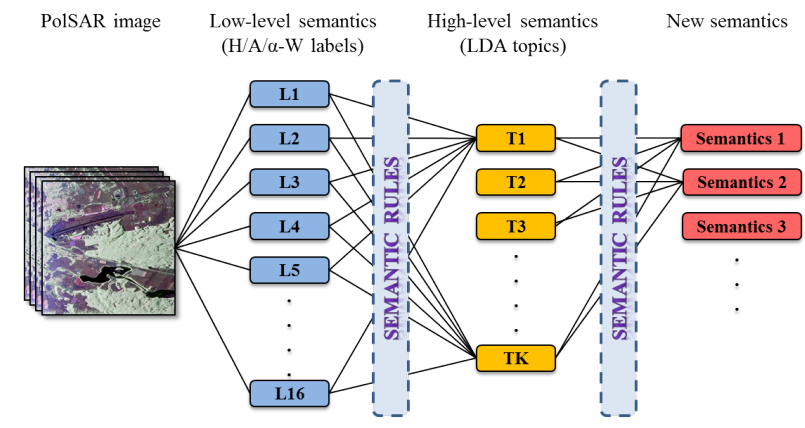

Fig. 1. Stepwise low- to high-level semantics generation.

remote sensing image type, namely Polarimetric Synthetic Aperture Radar (PolSAR) images, closer to human semantics. This helps computers to discover existing semantic relationships within images and employ them for analyzing user queries (which are based on semantics) and provide them with semantically meaningful relevant results. Fig. 1 shows an overview of the proposed approach.

PolSAR images supply information with respect to the physical scattering properties of the recorded ground targets, retrieved by applying coherent or incoherent target decomposition theorems to the first- and second-order polarimetric representations [6]. A widely employed decomposition method is Entropy/Anisotropy/Alpha $(\mathrm{H} / \mathrm{A} / \alpha)$, resulting in three parameters describing the physics behind the scattering processes. These parameters lead to a superior pixel-based unsupervised classification scheme, the $\mathrm{H} / \mathrm{A} / \alpha$ classification method [7]. A modification to this method, namely the $H / A / \alpha$-Wishart classification method [8], shows that the complex Wishart distribution parameters improve the classification substantially.

In our proposed approach, we employ the $\mathrm{H} / \mathrm{A} / \alpha$-Wishart method for discovering the low-level semantics of PolSAR images as a set of classes, representing targets by their physical scattering properties (e.g., low-entropy surface scattering with high anisotropy). The images are then tiled into patches, and each image patch is modeled as a Bag-of-Words (BoW) by generating a histogram of its assigned class labels, where the labels are the words in the BoWs (see Fig. 2). After that, a generative statistical model, namely Latent Dirichlet Allocation (LDA) [9], is applied to the BoW histograms in order to discover the latent semantics behind the image patches as a set of topics. Our validation of the topics based on ground truth (Google Earth ${ }^{1}$ ) images demonstrates that they provide

\footnotetext{
${ }^{1}$ https://www.google.com/earth/
} 
high-level semantics which are close to human semantics used for identifying land-cover types (e.g., woody vegetation).

As the topics are the basic land-cover types existing in the image patches, further land-cover types can be defined as combinations of the basic land-cover types (e.g., a shoreline is a combination of a water body, grassland, and woody vegetation). Thus, the image patches can be modeled by vectors of topic mixtures, the co-called Bag-of-Topics (BoT) model [10]. The topic vectors form a multi-dimensional Euclidean space in which each image patch is represented as a point. Since the dimensions of this space are semantically meaningful, it can be easily explored and assessed through immersive visualization techniques to discover existing semantic relationships and identify new semantic categories (e.g., mixed land-cover types). While a topic representation of images can adapt computer image interpretation to human semantics, the semantic relationships can be used for designing rule-based land-cover categorization methods.

Section II reviews H/A/ $\alpha$-Wishart classification. Section III describes low-level semantics discovery. Section IV briefly introduces LDA. Section V and VI explain how to discover high-level semantics and explore it for finding new semantics. Section VII concludes the paper.

\section{ENTROPY/ANISOTROPY/ALPHA-WiSHART CLASSIFICATION}

Our test image is an F-SAR airborne complex-valued dataset $^{2}$ comprising four polarization planes $(\mathrm{VV}, \mathrm{HH}, \mathrm{HV}$, and $\mathrm{VH}$ ) [11]. The data were multi-looked with a factor of 5 in azimuth direction. In order to reduce the inherent speckle noise, PolSAR data are usually delivered in a multi-looked pixel-wise coherency matrix format $T$. This matrix is a $3 \times 3$ Hermitian, positive, and semi-definite matrix which can be written as:

$$
T=U \cdot \Sigma \cdot U^{-1},
$$

where $\Sigma$ is a diagonal matrix composed of the eigenvalues of $T$ ( $\lambda_{1}, \lambda_{2}$ and $\lambda_{3}$ in descending order). The columns of $U$ contain the corresponding eigenvectors to the eigenvalues $\left(u_{1}\right.$, $u_{2}$ and $u_{3}$ ), where each $u_{i}$ can be further decomposed into [6]:

$$
u_{i}=\left[\begin{array}{lll}
\cos \alpha_{i} & \sin \alpha_{i} \cos \beta_{i} e^{j \delta_{i}} & \sin \alpha_{i} \cos \beta_{i} e^{j \gamma_{i}}
\end{array}\right]^{T} .
$$

From Eq. 1 and 2 the following parameters can be computed:

$$
H=\sum_{i=1}^{3}-p_{i} \log _{3} p_{i}, \quad A=\frac{p_{2}-p_{3}}{p_{2}+p_{3}}, \quad \alpha=\sum_{i=1}^{3} p_{i} \alpha_{i},
$$

where $p_{i}=\lambda_{i} / \sum_{j=1}^{3} \lambda_{j}$ denotes the probability of the eigenvalues $\lambda_{i}$. These parameters refer to the physics behind the scattering processes. The entropy $H$ discriminates pure and distributed scatterers, the anisotropy $A$ characterizes different types of scattering [6], and the mean $\alpha$ angle shows the dominant scattering mechanism,

Combinations of these parameters can lead to very good classification schemes. For example, the scheme in Fig. 2 divides the $H / \alpha$ plane into nine zones (classes), from which

${ }^{2}$ courtesy of Rolf Scheiber from DLR's Microwaves and Radar Institute

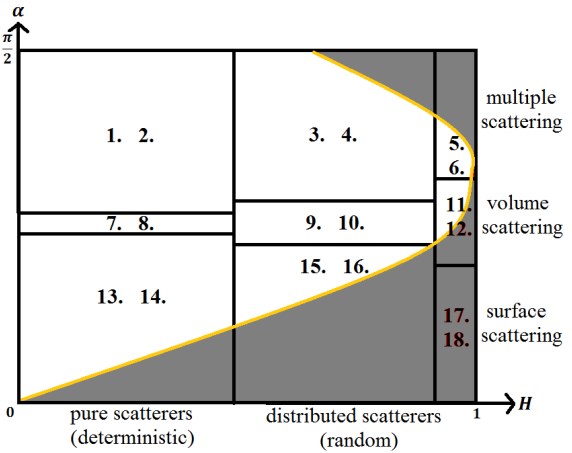

Fig. 2. The $H / \alpha$ classification scheme (adapted from [7]). From the 18 classes, only 1-16 are feasible and are assigned as labels L1-L16 in Table I.

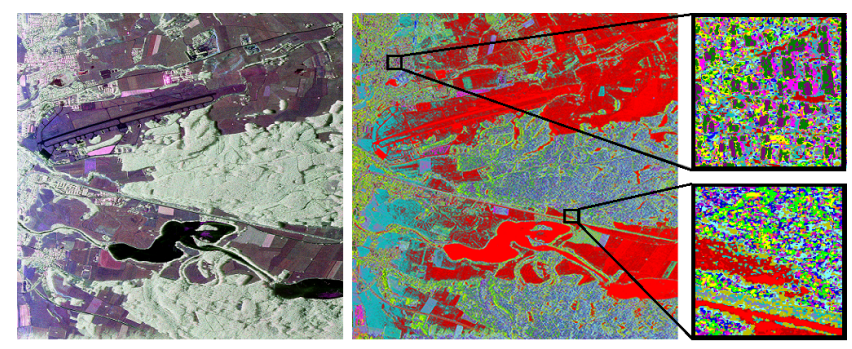

Fig. 3. False-color Pauli RGB representation of our test image (left). H/A/ $\alpha$ Wishart classification map together with two zoomed-in subareas (right), where the colors correspond to the low-level semantics in Table I.

only eight are feasible. Including anisotropy will double the number of classes: nine for $A \leq 0.5$ (denoted by odd labels) and nine for $A>0.5$ (denoted by even labels). The $H / A / \alpha$ classification method has its own drawbacks such as the fixed boundaries of the classes, which do not dynamically adapt to the input data. Dealing with this issue, the authors of [8] proposed to use the parameters of the complex Wishart distribution of the coherency matrix. This approach employs the $H / A / \alpha$ classification for initializing the classes, and then uses the Wishart parameters in an iterative procedure to refine the classification. Its main advantages are to consider the magnitude of the coherency matrix, which is important in detecting surface scattering, and allowing a dynamic adaptation of the class boundaries to the input data.

\section{LOW-LeVEL SEMANTICS Discovery}

We apply the $H / A / \alpha-W i$ shart classification, based on $5 \times 5$ pixel windows, to our PolSAR test image, shown in Fig. 3 (left), in order to retrieve a classification map with 16 classes. The class labels are then considered as low-level semantics, which refer to the scattering properties of the recorded targets. Fig. 3 (right) shows the classification results, with Table I defining the color coding.

The labels in Table I can be categorized into three main scattering mechanisms: the first six labels $(L 1-L 6)$ refer to Multiple Scattering (MS), which usually occur in urban and forested areas. The next six labels $(L 7-L 12)$ refer to Volume Scattering (VS) which is usually observed in forested and vegetated areas, while the labels $L 13-L 16$ refer to Surface Scattering (SS) which can be usually seen on rough land surfaces. These scattering mechanisms can be further divided into several subcategories depending on $\mathrm{H}$, which hints to the 
TABLE I

LOW-LEVEL SEMANTICS

\begin{tabular}{|c|l|}
\hline Label & \multicolumn{1}{|c|}{ Semantics } \\
\hline L1 & Low-entropy multiple scattering with low anisotropy \\
L2 & Low-entropy multiple scattering with high anisotropy \\
\hline L3 & Medium-entropy multiple scattering with low anisotropy \\
\hline L4 & Medium-entropy multiple scattering with high anisotropy \\
\hline L5 & High-entropy multiple scattering with low anisotropy \\
\hline L6 & High-entropy multiple scattering with high anisotropy \\
L7 & Low-entropy volume scattering with low anisotropy \\
L8 & Low-entropy volume scattering with high anisotropy \\
L9 & Medium-entropy volume scattering with low anisotropy \\
L10 & Medium-entropy volume scattering with high anisotropy \\
L11 & High-entropy volume scattering with low anisotropy \\
L12 & High-entropy volume scattering with high anisotropy \\
\hline L13 & Low-entropy surface scattering with low anisotropy \\
L14 & Low-entropy surface scattering with high anisotropy \\
L15 & Medium-entropy surface scattering with low anisotropy \\
L16 & Medium-entropy surface scattering with high anisotropy \\
\hline
\end{tabular}

number of scatterers being present in each resolution cell, and on A, showing the importance of the secondary scatterers.

\section{LATENT DiRichlet AllocAtion}

A widely used statistical generative model for the discovery of hidden semantic structures behind collections of images is Latent Dirichlet Allocation (LDA) [9]. Assuming each image $\mathbf{w}_{d}$, as a combination of $N_{d}$ visual word-tokens, $\mathbf{w}_{d}=$ $\left\{w_{d 1}, w_{d 2}, \ldots, w_{d N_{d}}\right\}$, LDA discovers its latent semantics as a set of $k$ topics, where the topics are distributions over a fixed dictionary of $N_{V}$ visual words. They are supposed to reflect semantic categories. Therefore, each image containing different targets is represented as a mixture of the topics.

For estimating the required model parameters $a$ and $B$, and inferring the posterior distributions (i.e., the topic distributions in the images), LDA uses approximate inference algorithms such as variational Expectation Maximization [9].

Having the model parameters and the posterior distributions, LDA can generate every $n$-th visual word-token $\left(w_{d n}\right)$ of each image $\mathbf{w}_{d}$ through the generative process:

$p\left(w_{d n} \mid a, B\right)=\int p\left(\theta_{d} \mid a\right)\left(\sum_{z_{d n}} p\left(z_{d n} \mid \theta_{d}\right) p\left(w_{d n} \mid z_{d n}, B\right)\right) d \theta_{d}$

In this process, LDA chooses a $k$-dimensional Dirichlet random variable $\theta_{d} \sim \operatorname{Dir}(a)$, corresponding to $\mathbf{w}_{d}$, where $a$ determines the Dirichlet distribution's prior [9]. Then it selects a topic-token $z_{d n}$ from the topic mixture $\theta_{d}$ and picks $w_{d n}$ from the multinomial probability distribution conditioned on the selected topic, $p\left(w_{d n} \mid z_{d n}, B\right)$. The matrix $B_{N_{V} \times k}$ parameterizes the visual word probabilities within the topics.

\section{High-Level Semantics Discovery}

Since the low-level semantics only refer to physical scattering properties, it is difficult to associate them with common land-cover categories. Therefore, in this section we employ LDA to discover higher-level semantics. To this end, we split each image into adjacent patches of $64 \times 64$ pixels and represent each image patch as a BoW by generating a histogram of the 16 labels in Table I assigned to that image patch; the selected patch size of $64 \times 64$ pixels resulted as a compromise between small patches keeping the semantic analysis simple
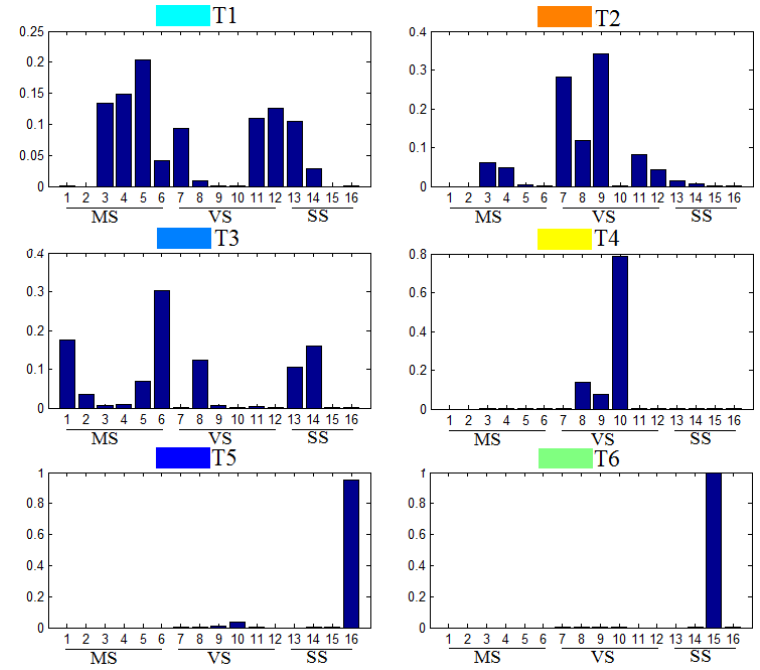

Fig. 4. Topics discovered by LDA: the $x$ axis denotes the 16 labels in Table I (e.g., $1 \rightarrow L 1$ ), while the $y$ axis shows their probabilities.
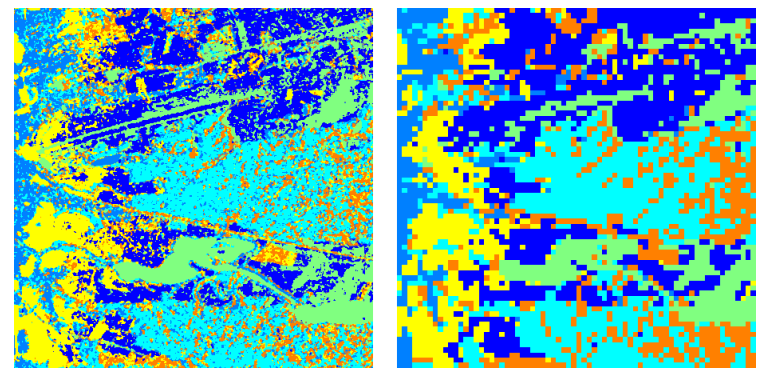

Fig. 5. Topic assignments to image pixels (left) and to image patches (right).

and bigger patches capturing the spatial context of objects. By considering each image patch as a document, we apply LDA to the BoW models to find latent semantics as a set of topics. In our experiments, the number of topics to be discovered was set to six based on an educated guess. The resulting topics are shown in Fig. 4 as probability distributions over the 16 labels. Fig. 5 shows the topic assignments to each image pixel and patch, where the assignments were made according to:

$$
\begin{aligned}
& \text { word } i \rightarrow \arg \left\{\max \left[p\left(w_{i} \mid z_{k}\right)\right]\right\}, \\
& \text { patch } j \rightarrow \arg \left\{\max \left[p\left(z_{k} \mid \theta_{j}\right)\right]\right\} .
\end{aligned}
$$

By analyzing Fig. 4 and 5, and by using precisely coregistered Google Earth images as correct ground truth, it is possible to reveal the semantics of the topics and the rules connecting them to the lower-level semantics. As shown in Table II, the probabilities of the respective types of scattering can be written as percentages:

TABLE II

High-LeVEl SEMANTiCS

\begin{tabular}{|c|l|c|}
\hline Topic & \multicolumn{1}{|c|}{ Semantics } & \multicolumn{1}{c|}{ Semantic rule } \\
\hline T1 & Woody vegetation & $53 \% \mathrm{MS}+34 \% \mathrm{VS}+13 \% \mathrm{SS}$ \\
T2 & Mixed woody vegetation \& shrubs & $12 \% \mathrm{MS}+86 \% \mathrm{VS}+02 \% \mathrm{SS}$ \\
T3 & Artificial, man-made structures & $60 \% \mathrm{MS}+14 \% \mathrm{VS}+26 \% \mathrm{SS}$ \\
T4 & Herbaceous vegetation & $00 \% \mathrm{MS}+100 \% \mathrm{VS}+00 \% \mathrm{SS}$ \\
T5 & Smooth surface & $00 \% \mathrm{MS}+05 \% \mathrm{VS}+95 \% \mathrm{SS}$ \\
T6 & Specular surface & $00 \% \mathrm{MS}+00 \% \mathrm{VS}+100 \% \mathrm{SS}$ \\
\hline
\end{tabular}




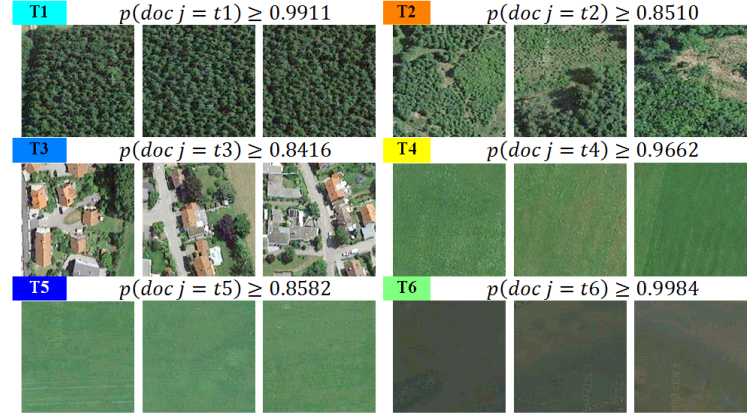

Fig. 6. Visualization of the discovered topic semantics. While discriminating the image patches of T4 and T5 is difficult due to their similar colors, they could be separated by using PolSAR images based on their different dominant scattering mechanisms (VS vs. SS) caused by the distinct vegetation height.

- $T 1$ is mostly assigned to forested regions. The high MS and VS values are due to the presence of trunks and rich canopies of woody vegetation. In addition, the low SS value may be caused by canopy tops or ground. According to Fig. 4, T1's main component is L5, which is referred to in [7] as double-bounce scattering in a high-entropy environment, like vegetation which has a well developed branch and crown structure. Therefore, we assign the semantic term woody vegetation to T1.

- $T 2$ is most often assigned to regions covered by trees and shrubs. The very small SS value indicates the absence of rich canopies which allows radar waves to scatter directly back to the sensor. The relatively large MS value shows the small number of tree trunks. T2's main component is L9 which [7] refers to vegetated surfaces with anisotropic scatterers and moderate correlation of scatterer orientations. Thus, we assign the semantic term mixed woody vegetation and shrubs to $\mathrm{T} 2$.

- T3 is mostly characterized by MS caused by building walls and tree trunks. Additionally, VS and SS in this topic can be caused by tree canopies and smooth surfaces (e.g., streets, roofs), respectively. T3 is the only topic with non-zero L1 and L2. These are referred to in [7] as double bounce scattering events provided by isolated dielectric and metallic dihedral scatterers. Therefore, we assign the semantic term artificial/man-made structures to T3.

- T4 is characterized only by VS indicating that it usually occurs in regions covered by vegetation with thin stems which do not backscatter the incident wave. Thus, we assign the semantic term herbaceous vegetation to T4.

- T5 is distinguished by a very large SS and a very small VS value. This reveals thin land-cover allowing radar waves to reach the ground. Therefore, we assign the semantic term smooth surface to T5.

- T6 is completely composed of SS. Therefore, we assign the semantic term specular surface to it.

Fig. 6 demonstrates the ground truth (Google Earth images) of some image patches, which are mainly characterized by a single topic, in order to visualize the semantics of each topic.

\section{Assessment of High-Level Semantics}

In this section, we assess the discovered high-level semantics by modeling each image patch as a vector of the extracted

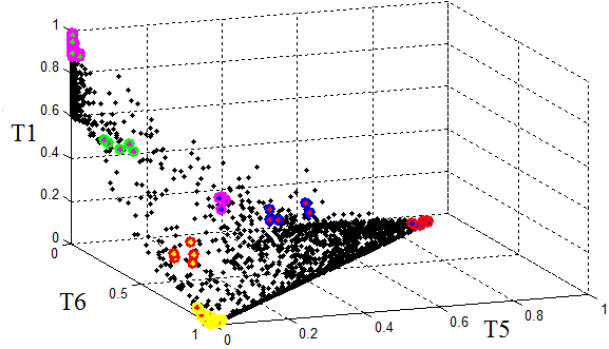

Fig. 7. The three-dimensional space spanned by T5, T6, and T1. The highlighted points are visualized in Fig. 8.

topics (BoT model). These vectors form a multi-dimensional Euclidean space, the so-called topic space, in which each image patch is represented as a point positioned according to its topic distribution. Since the topic space dimensions are semantically meaningful (e.g., woody vegetation), the image patch distribution within this space is easily understandable. When these topics are the basic land-cover types, different topic mixtures result in various mixed land-cover types. Thus, by navigating through the topic space, users can easily reach the image patches containing their desired land-cover types and define new categories of mixed land-cover types.

In order to visualize topic spaces, we select three topics and pick up the image patches containing them. After discarding the other topics, the chosen image patches are displayed in a three-dimensional normalized topic space. We explore the topic space and pick groupings of image patches from different regions and validate their semantics via their ground truth.

\section{A. Natural Land-Cover Variation}

Our aim is to discover different natural land-cover types as mixtures of the basic land-cover types: woody vegetation (T1), smooth land surfaces (T5), and specular surfaces (T6). Fig. 7 shows the topic space of these topics in which the coordinates are $(T 1, T 5, T 6)$. Here, the selected image patches are highlighted and their ground truth is shown in Fig. 8.

In Fig. 7, starting from the point $(0,0,1)$, we expect to have image patches of specular surfaces such as water bodies which complies with Fig. 8 (grouping G1). By moving toward the point $(1,0,0)$, the proportion of the water bodies decreases while that of the forest increases, as demonstrated in Fig. 8 (G2) and (G3). Reaching the point $(1,0,0)$, the image patches are totally covered by forest, as shown in Fig. 8 (G4). Furthermore, around the point $(0.3,0.3,0.3)$ a mixture of the three basic land-cover types is expected which is true according to Fig. 8 (G5). By moving toward the point $(0,1,0)$ the land-cover types change, containing higher proportions of grasslands as shown in Fig. 8 (G6) and (G7).

\section{B. Natural and Man-made Land-Cover Variation}

The idea is to discover various natural and man-made landcover types as mixtures of the basic land-cover types: mixed woody vegetation and shrubs (T2), man-made structures (T3), and smooth surfaces (T5). Fig. 9 shows the topic space of the image patches in which the coordinates are $(T 2, T 3, T 5)$.

As shown in Fig. 9, we selected three groupings of image patches from various regions: one grouping around the location 


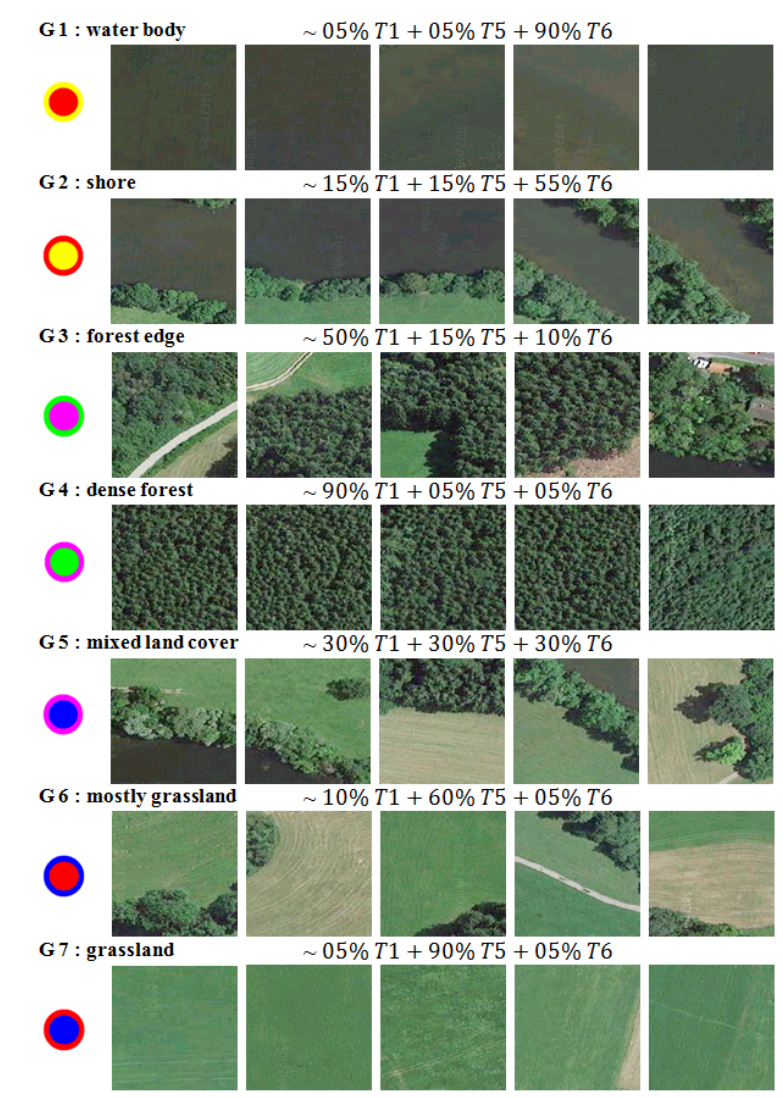

Fig. 8. Examples of new semantic classes spanned by T5, T6 and T1 corresponding to the highlighted points in Fig. 7.

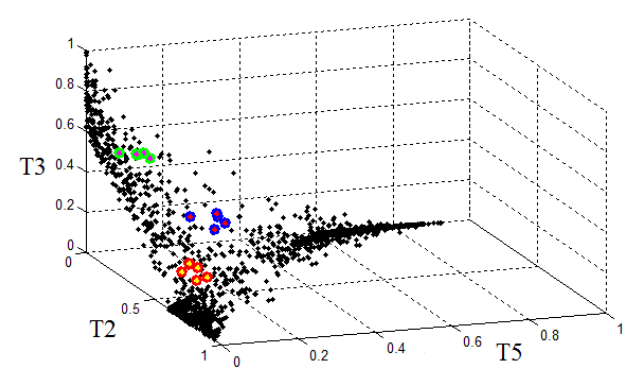

Fig. 9. The three-dimensional topic space spanned by T5, T2, and T3. The highlighted points are visualized in Fig. 10.

$(0.5,0.1,0.1)$, one grouping around the location $(0.3,0.3,0.3)$, and one grouping around the location $(0.1,0.6,0.1)$. The visualization of their ground truth in Fig. 10 indicates that the image patches of the first grouping are mostly covered by sparse forest. The image patches of the second grouping contain mixtures of all the three basic land-cover types, while the third grouping mostly represents residential areas including man-made structures (e.g., houses) and trees.

Altogether, representing images by their high-level semantics helps us to better understand their contents and discover new semantic categories as mixtures of high-level semantics. This further allows us to categorize images by selecting a set of coordinates and to partition the topic space.

\section{CONCLUSION}

In this letter, we propose a multi-level approach for semantic relationship discovery in PolSAR images. First, we extract

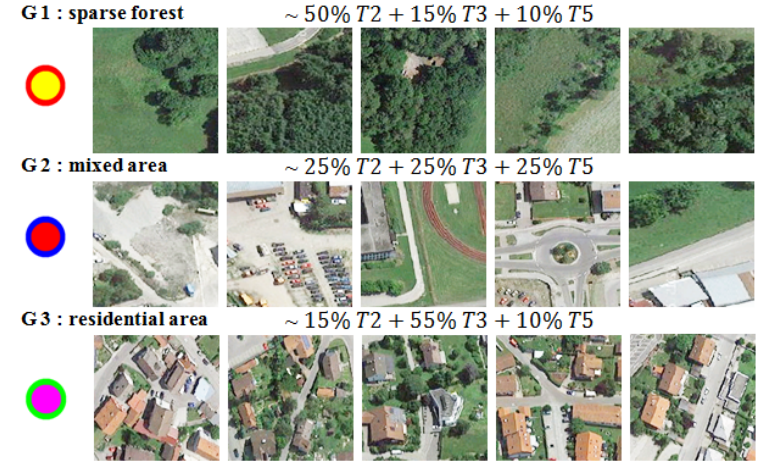

Fig. 10. Examples of new semantic classes spanned by T5, T2, and T3 corresponding to the highlighted points in Fig. 9.

low-level semantics (physical properties of the recorded targets) by using the $H / A / \alpha-W$ ishart classification method. Then, the images are tiled into patches and modeled as BoWs by generating histograms of the class-labels assigned to the image patches. Next, LDA is applied to the BoWs to discover higher-level semantics as a set of topics. Our analysis shows that the topics refer to basic land-cover types and the image patches covered by other land-cover types (e.g., mixed landcover types) are described as mixtures of the topics. Thus, modeling image patches by the topics (BoT) helps to better understand the existing semantic relationships and identify new land-cover type categories. The semantic relationships can be used in designing parameter-based land-cover categorization methods. Moreover, a topic representation of PolSAR images helps image mining systems to adapt their results to human semantics, thus narrowing the semantic gap.

\section{REFERENCES}

[1] S. S. Durbha and R. L. King, "Semantics-enabled framework for knowledge discovery from Earth observation data archives," IEEE TGRS, vol. 43, pp. 2563-2572, Nov. 2005.

[2] M. Datcu, H. Daschiel, A. Pelizzari, M. Quartulli, A. Galoppo, A. Colapicchioni, M. Pastori, K. Seidel, P. G. Marchetti, and S. D'Elia, "Information mining in remote sensing image archives: System concepts," IEEE TGRS, vol. 41, pp. 2923-2936, Dec. 2003.

[3] K. Alonso and M. Datcu, "Accelerated probabilistic learning concept for mining heterogeneous Earth observation images," IEEE JSTARS, vol. 8, pp. 3356-3371, Jul. 2015.

[4] R. Bahmanyar, A. Murillo Montes de Oca, and M. Datcu, "The semantic gap: An exploration of user and computer perspectives in Earth observation images," IEEE GRSL, vol. 12, pp. 2046-2050, Oct. 2015.

[5] D. Bratasanu, I. Nedelcu, and M. Datcu, "Bridging the semantic gap for satellite image annotation and automatic mapping applications," IEEE JSTARS, vol. 4, pp. 193-204, Mar. 2011.

[6] S. R. Cloude and E. Pottier, "A review of target decomposition theorems in radar polarimetry," IEEE TGRS, vol. 34, pp. 498-518, Mar. 1996.

[7] — "An entropy based classification scheme for land applications of polarimetric SAR," IEEE TGRS, vol. 35, pp. 68-78, Jan. 1997.

[8] J.-S. Lee, M. R. Grunes, T. L. Ainsworth, L.-J. Du, D. L. Schuler, and S. R. Cloude, "Unsupervised classification using polarimetric decomposition and the complex Wishart classifier," IEEE TGRS, vol. 37, pp. 2249-2258, Sep. 1999.

[9] D. M. Blei, A. Y. Ng, and M. I. Jordan, "Latent Dirichlet Allocation," $J M L R$, vol. 3, pp. 993-1022, Jan. 2003.

[10] R. Bahmanyar, S. Cui, and M. Datcu, "A comparative study of Bag-ofWords and Bag-of-Topics models of EO image patches," IEEE GRSL, vol. 12, pp. 1357-1361, Jun. 2015.

[11] R. Horn, A. Nottensteiner, A. Reigber, J. Fischer, and R. Scheiber, "FSAR - DLR's new multifrequency polarimetric airborne SAR," in IEEE IGARSS, vol. 2, Jul. 2009, pp. 902-905. 